\section{Dynamic noninvasive monitoring of renal function in vivo by fluorescence lifetime imaging}

\author{
Reece J. Goiffon, ${ }^{a}$ Walter J. Akers, ${ }^{a}$ Mikhail Y. Berezin, ${ }^{a}$ \\ Hyeran Lee, $^{\mathrm{a}}$ and Samuel Achilefu $\mathrm{u}^{\mathrm{a}, \mathrm{b}, *}$ \\ a'Washington University School of Medicine, Department of \\ Radiology, St. Louis, Missouri 63110 \\ ${ }^{b}$ Washington University School of Medicine, Department of \\ Biochemistry and Molecular Biophysics, St. Louis, \\ Missouri 63110
}

\begin{abstract}
Kidneys normally filter the blood of excess salts and metabolic products, such as urea, while retaining plasma proteins. In diseases such as multiple myeloma and diabetes mellitus, the renal function is compromised and protein escapes into the urine. In this study, we present the use of fluorescence lifetime imaging (FLI) to image excess serum protein in urine (proteinuria). The near-infrared fluorescent dye LS-288 has distinct lifetimes when bound to protein versus free in solution, providing contrast between the protein-rich viscera and the mostly protein-free bladder. FLI with LS-288 in mice revealed that fluorescence lifetime (FLT) differences in the bladder relative to surrounding tissues was due to the fractional contributions of the bound and unbound dye molecules. The FLT of LS-288 decreased in the case of proteinuria while fluorescence intensity was unchanged. The results show that FLI can be useful for the dynamic imaging of proteinlosing nephropathy due to diabetes mellitus and other renal diseases and suggest the potential use of the FLI to distinguish tumors from fluid-filled cysts in the body.

() 2009 Society of Photo-Optical Instrumentation Engineers. [DOI: $10.1117 / 1.3095800]$
\end{abstract}

Keywords: optical imaging; fluorescence lifetime; near-infrared; proteinuria; albumin; nephropathy.

Paper 08277LR received Aug. 8, 2008; revised manuscript received Nov. 26, 2008; accepted for publication Jan. 10, 2009; published online Apr. 13, 2009.

\section{Introduction}

Proteinuria (PU), the presence of abnormally high levels of protein in urine, is a marker of chronic kidney disease (CKD), which can lead to end-stage renal disease. ${ }^{1}$ A recent study has shown that $11 \%$ of Americans over 20 years of age have CKD and $63 \%$ of these have microalbuminuria. ${ }^{2}$ Early detection of kidney dysfunction can be useful in treating diabetes mellitus, urinary tract infections, polycystic kidney disease, autoimmune diseases, transplant rejection, ${ }^{3}$ and acute toxicity. $^{4}$

Nuclear imaging has been a mainstay of urology for years but remains expensive, hazardous, and time consuming. Op-

*Tel. 314-362-8599; Fax: 314-747-5191; E-mail: achilefus@mir.wustl.edu.

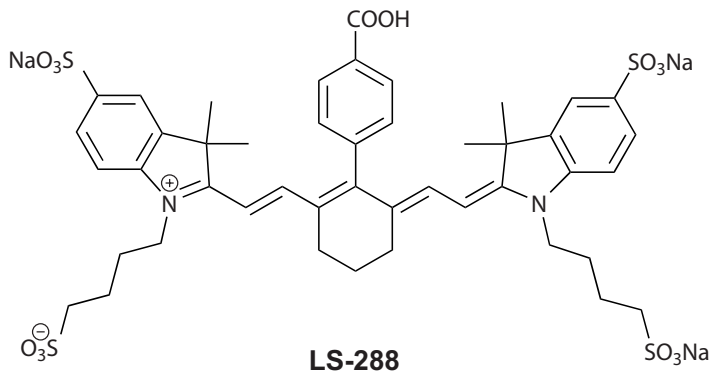

Fig. 1 Structure of LS-288 used as fluorescence lifetime reporter in the bladder.

tical imaging using near-infrared (NIR) fluorescent contrast agents is an alternative and a highly sensitive method for imaging living systems. For example, indocyanine green has been used to assess liver function, breast cancer detection, or lymph node mapping. ${ }^{5,6}$ Although fluorescence intensity measurements report molecular processes with high sensitivity, the fluorescence lifetime (FLT) of dyes provides complementary information that is less dependent on the dye concentration. In particular, fluorescence lifetime imaging (FLI) can provide a wide range of information about the microenvironment of the dye molecules such as $\mathrm{pH}$, ionic concentration, oxygen saturation, and temperature. ${ }^{7-9}$ Previously, we demonstrated that FLT of polymethine dyes are sensitive to medium polarity and may change upon binding to proteins. ${ }^{10}$ Urine in the bladder is a unique environment in the abdomen because it is largely free of protein in healthy individuals. Therefore, dyes that have distinct FLTs in protein-rich and free (aqueous) environments could serve as dynamic FLT reporters of proteinuria.

In this study, we demonstrate that FLI can be used to continuously image the functional status of the kidneys and bladder by using a protein-sensitive NIR fluorescent dye, LS-288. ${ }^{11}$ Our results show that changes in the fractional contributions of two distinct FLTs of LS-288 correlate with free and protein bound states, thereby providing dynamic FLI of $\mathrm{PU}$ in mice.

\section{Methods}

All animal studies were performed in compliance with the Washington University in St. Louis School of Medicine Animal Studies Committee requirements using male NCR nude mice. A $100 \mu \mathrm{L}$ injection of $1.0 \mathrm{mg} / \mathrm{mL}$ bovine serum albumin (BSA) in PBS was administered transcutaneously into the bladder in mice to simulate PU. The control group did not receive an injection to prevent contamination of urine with protein. All mice received $6 \mathrm{nmol}$ of LS-288 (Fig. 1) ${ }^{11}$ via the lateral tail vein prior to imaging. Mouse images were acquired with a time-domain diffuse optical imaging system (eXplore OptixART, Montreal, Canada) and data were analyzed as described previously. ${ }^{7,12}$ Bladder regions were identified by gating to an appropriate monoexponential FLT to remove pixels surrounding the bladder region (below $1.0 \mathrm{~ns}$ for control and $1.1 \mathrm{~ns}$ for PU mice). Biexponential FLT maps for bladder and control areas were generated for quantification.

1083-3668/2009/14(2)/020501/3/\$25.00 @ 2009 SPIE 


\section{JBO LETTERS}

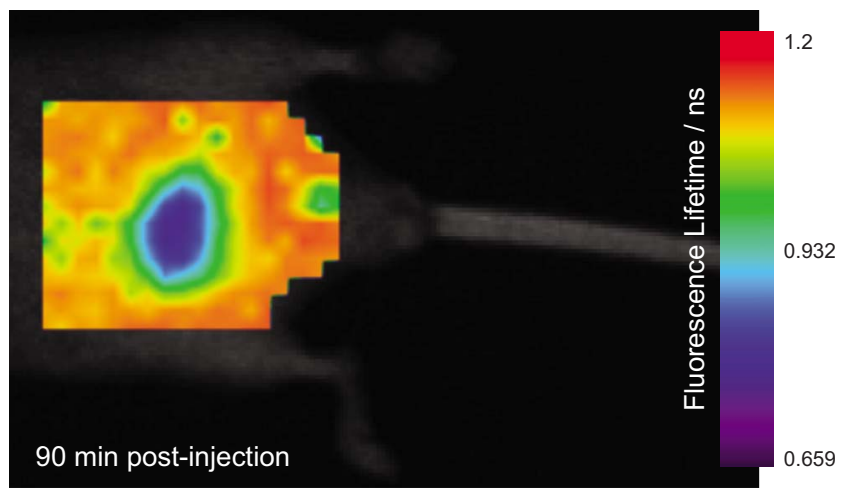

Fig. 2 FLT map of mouse abdomen acquired 90 min after injection of $6 \mathrm{nmol}$ LS-288 IV. The low FLT region in the center of the abdomen is the filled urinary bladder. Video 1 Single FLT map from scans of the abdominal region taken in series over $4 \mathrm{~h}$. The video shows the bladder growing at a steady rate followed by a period characterized by a nearly constant bladder size and a change in FLT around the penis as urine leaks onto the skin and dries. (QuickTime, 0.9 MB). [URL: http://dx.doi.org/10.1117/1.3095800.1]

\section{Results and Discussion}

\subsection{Dynamic Bladder Imaging in Real Time}

We used LS-288 for this study because it has distinctly different FLTs when free versus interacting with proteins in solution. ${ }^{13}$ Using a series of FLT map images, the change in bladder size was visualized over time (Fig. 2 and Video 1). FLI provided excellent contrast between the low-protein urine in the bladder and the surrounding protein-rich tissues. In ad- dition to imaging the bladder and abnormalities in the region, this method could be used to monitor urine flow rate or frequency of urination without invasive procedures such as surgery or urethral catheterization.

\subsection{Fluorescence Lifetime Data Analysis}

A single exponential fitting of the TPSF decay provides a single value $\bar{\tau}$, which can be considered a weighted average of the values obtained through biexponential fit corresponding to the unbound $\left(\tau_{1}\right)$ and protein-bound $\left(\tau_{2}\right)$ fractions $\left(f_{1}\right.$ and $f_{2}$, respectively) of LS-288 [Eq. (1)]. ${ }^{13}$ The measured values of $\tau_{1}$ and $\tau_{2}$ for LS-288 remained relatively constant after intravenous administration in living mice despite a large change in $\bar{\tau}$ (Table 1). Dye molecules in the blood and other tissues are largely protein bound $\left(\bar{\tau} \approx \tau_{2}\right)$, with only slight contribution from $\tau_{1}$, as demonstrated by FLT gating of fluorescence intensity values for each FLT from the biexponential model mapped over the bladder (Fig. 3). Thus, the single exponential fitting of FLI data can be used to simplify analysis and data representation [Fig. 3(c)]. FLT of the unbound LS-288 shown in Table 1 is closer to the lifetime of the dye in water $(0.44 \mathrm{~ns}){ }^{14}$

$$
\bar{\tau}=f_{1} \tau_{1}+f_{2} \tau_{2} .
$$

\subsection{Detection of Proteinuria In Vivo}

Variations in $\bar{\tau}$ values are expected between the individual PU mice because each mouse is hydrated differently, has a different metabolic status, and urinates at different times. The BSA

(a)

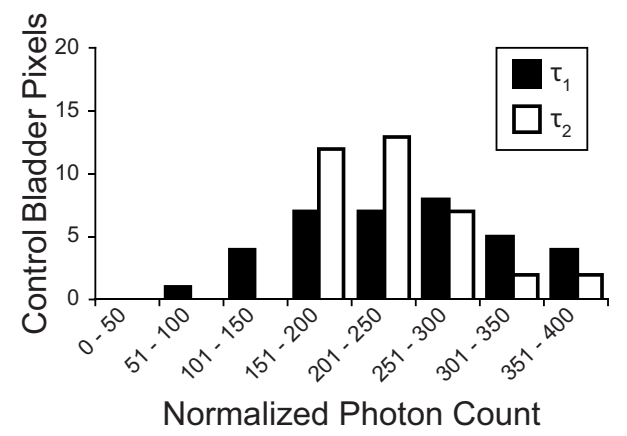

(b)
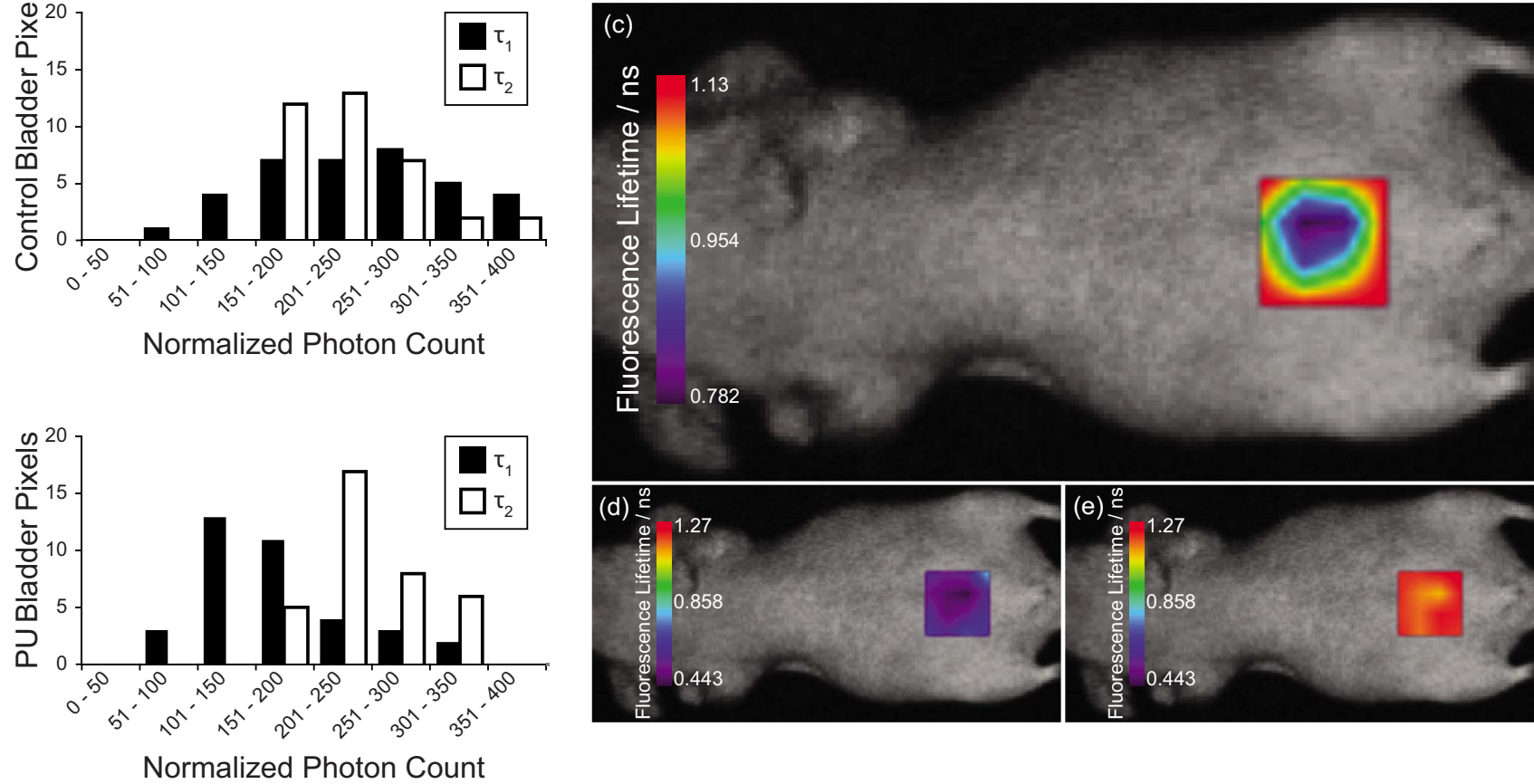

Fig. 3 Fluorescence lifetime-gated imaging of mouse bladders. Histograms show the relative emission intensities of $\tau_{1}=0.595 \pm 0.092 \mathrm{~ns}$ and $\tau_{2}$ $=1.197 \pm 0.040 \mathrm{~ns}$ from biexponential fitting of fluorescence decay profiles in (a) control and (b) PU mice $(n=4)$. The difference in $\bar{\tau}$ is due to the change in contribution from unbound $\left(\tau_{1}\right)$ relative to bound $\left(\tau_{2}\right)$. (c) $\bar{\tau}$ varies by $0.34 \mathrm{~ns}$ from edge to center of a 144 -mm ${ }^{2}$ bladder despite relatively constant values of (d) $\tau_{1}$ and (e) $\tau_{2}$. These analyses demonstrate that the change in $\bar{\tau}$ is due to change in the fractional intensity contribution from bound and unbound dye, not by change in the values of $\tau_{1}$ or $\tau_{2}$. 


\section{JBO LETTERS}

Table 1 FLT values from bladder and adjacent body regions of interest (ROIs) in normal and PU mice. Single exponential fitting of the fluorescence decay profiles $(\bar{\tau})$ from control and PU bladder ROls demonstrated significant difference, while $\tau_{1}$ and $\tau_{2}$ were not different between sets $(p>0.01)$. This shows that it is possible to detect abnormally high levels of protein in the urine by determining only $\bar{\tau}$, which corresponds to the fractional contribution of bound and unbound dye molecules $\left(f_{1} / f_{2}\right)$.

\begin{tabular}{lcccc}
\hline & $\bar{\tau} / \mathrm{ns}$ & $\tau_{1} / \mathrm{ns}$ & $\tau_{2} / \mathrm{ns}$ & $f_{1} / f_{2}$ \\
\hline Control bladders & 0.682 & 0.451 & 0.966 & 1.07 \\
& $\pm 0.029^{a}$ & \pm 0.034 & \pm 0.084 & \pm 0.38 \\
PU bladders & 0.794 & 0.427 & 1.084 & 0.69 \\
& $\pm 0.050^{a}$ & \pm 0.043 & \pm 0.062 & \pm 0.21 \\
Control bodies & 0.90 & 0.465 & 1.061 & 0.43 \\
& \pm 0.12 & \pm 0.050 & \pm 0.066 & \pm 0.33 \\
PU bodies & 1.037 & 0.510 & 1.172 & 0.22 \\
& \pm 0.078 & \pm 0.076 & \pm 0.042 & \pm 0.13 \\
\hline
\end{tabular}

${ }^{a}$ Denotes $p<0.01$

solution concentration of $1.0 \mathrm{mg} / \mathrm{mL}$ used in this study corresponds with macroalbuminuria, ${ }^{15}$ but the combination of continuous glomerular filtration, varying initial urine volume, and sporadic urination allows for a wider range of albumin levels to be examined. This is reflected in the PU results for individual mice, and despite the variability between each of them, the PU $\bar{\tau}$ values are significantly different from those of the control mice. In both treatment sets, the bladder was distinguishable from the body by using $\bar{\tau}$. Statistical analysis shows that there is a significant difference between PU and control bladders and between both bladder ROI sets and their respective body ROIs (Table 1 ).

\section{Conclusions}

NIR fluorescent dye-mediated FLI is an exciting optical imaging method with a great potential to unravel physiological and molecular processes in cells and living organisms. Particularly, the early detection of nephropathy due to diabetes mellitus and other causes would improve treatment for patients. Our results show that the FLI of mouse bladder with LS-288 allows for dynamic imaging of bladder physiology and proteinuria. Because the bladder is a superficial organ in humans, clinical translation of the FLI method using a mouse model of proteinuria described herein is feasible. The FLI strategy is applicable to other organs penetrable by NIR light to improve diagnostic information. For example, the distinct FLT behavior of LS-288 in different environments suggests the potential use of FLI to distinguish tumors from fluid-filled cysts in the body or to identify solid cancer in the bladder.

\section{Acknowledgments}

This study was funded, in part, by the National Institutes of Health (Grants No. R01 CA109754, No. R01 EB1430, and No. U01 HL080729).

\section{References}

1. F. Locatelli, L. D. Vecchio, and P. Pozzoni, "The importance of early detection of chronic kidney disease," Nephrol. Dial Transplant 17 (Suppl 11), 2-7 (2002).

2. J. Coresh, B. C. Astor, T. Greene, G. Eknoyan, and A. S. Levey, "Prevalence of chronic kidney disease and decreased kidney function in the adult US population: Third National Health and Nutrition Examination Survey," Am. J. Kidney Dis. 41, 1-12 (2003).

3. J. A. Vassalotti, L. A. Stevens, and A. S. Levey, "Testing for chronic kidney disease: a position statement from the National Kidney Foundation," Am. J. Kidney Dis. 50, 169-180 (2007).

4. V. S. Vaidya, M. A. Ferguson, and J. V. Bonventre, "Biomarkers of acute kidney injury," Annu. Rev. Pharmacol. Toxicol. 48, 463-493 (2008).

5. B. N. Giepmans, S. R. Adams, M. H. Ellisman, and R. Y. Tsien, "The fluorescent toolbox for assessing protein location and function," Science 312, 217-224 (2006).

6. J. Rao, A. Dragulescu-Andrasi, and H. Yao, "Fluorescence imaging in vivo: recent advances," Curr. Opin. Biotechnol. 18, 17-25 (2007).

7. S. Bloch, F. Lesage, L. McIntosh, A. Gandjbakhche, K. Liang, and S. Achilefu, "Whole-body fluorescence lifetime imaging of a tumortargeted near-infrared molecular probe in mice," J. Biomed. Opt. 10 , 054003 (2005).

8. D. Elson, J. Requejo-Isidro, I. Munro, F. Reavell, J. Siegel, K. Suhling, P. Tadrous, R. Benninger, P. Lanigan, J. McGinty, C. Talbot, B. Treanor, S. Webb, A. Sandison, A. Wallace, D. Davis, J. Lever, M. Neil, D. Phillips, G. Stamp, and P. French, "Time-domain fluorescence lifetime imaging applied to biological tissue," Photochem. Photobiol. Sci. 3, 795-801 (2004).

9. K. Suhling, P. M. French, and D. Phillips, "Time-resolved fluorescence microscopy," Photochem. Photobiol. Sci. 4, 13-22 (2005).

10. M. Y. Berezin, H. Lee, W. Akers, and S. Achilefu, "Near infrared dyes as lifetime solvatochromic probes for micropolarity measurements of biological systems," Biophys. J. 93, 2892-2899 (2007).

11. H. Lee, J. C. Mason, and S. Achilefu, "Heptamethine cyanine dyes with a robust C-C bond at the central position of the chromophore," J. Org. Chem. 71, 7862-7865 (2006).

12. W. Akers, F. Lesage, D. Holten, and S. Achilefu, "In vivo resolution of multiexponential decays of multiple near-infrared molecular probes by fluorescence lifetime-gated whole-body time-resolved diffuse optical imaging," Mol. Imaging 6, 237-246 (2007).

13. W. J. Akers, M. Y. Berezin, H. Lee, and S. Achilefu, "Predicting in vivo fluorescence lifetime behavior of near-infrared fluorescent contrast agents using in vitro measurements," J. Biomed. Opt. 13, 054042 (2008).

14. H. Lee, M. Y. Berezin, M. Henary, L. Strekowski, and S. Achilefu, "Fluorescence lifetime properties of near-infrared cyanine dyes in relation to their structures," J. Photochem. Photobiol., A 200, 438444 (2008).

15. P. E. de Jong and G. C. Curhan, "Screening, monitoring, and treatment of albuminuria: public health perspectives," J. Am. Soc. Nephrol. 17, 2120-2126 (2006). 